\title{
Use of adrenal arterial embolization in severe ACTH-dependent Cushing's syndrome
}

\author{
S.B. Blunt, M. Pirmohamed, V.K.K. Chatterjee, J.M. Burrin, D.J. Allison ${ }^{1}$ and \\ G.F. Joplin
}

Department of Medicine and 'Department of Diagnostic Radiology, Royal Postgraduate Medical School, Hammersmith Hospital, London W12 0NN, UK.

\begin{abstract}
Summary: The management of a patient with severe Cushing's syndrome due to ectopic ACTH produced by a medullary carcinoma of the thyroid is described. Initial treatment with maximal adrenolytic medical therapy and two attempts at bilateral adrenal venous infarction had failed to control the disease, and she was at that time unfit for surgery. Subsequent use of bilateral adrenal arterial embolization enabled medical therapy to produce sufficient control of the Cushing's syndrome to allow bilateral adrenalectomy to be performed successfully, with resultant cure of the excessive cortisol production.
\end{abstract}

\section{Introduction}

Adrenal venous infarction can occur inadvertently during diagnostic adrenal phlebography and may result in hypoadrenalism. ${ }^{1-3}$ The recognition of this as a complication has however resulted in some therapeutic benefit. Intentional bilateral venous infarction of the adrenals has been used as a novel form of treatment and palliation in patients with ACTH-dependent Cushing's syndrome who have been unsuitable for, or who have proved resistant to, other forms of treatment. ${ }^{4,5}$ Our group used this approach to control a very advanced case where only one adrenal had been removable by the initial operation; ${ }^{6}$ the method has also been used in the treatment of a frail patient with Conn's syndrome due to an aldosteronoma. ${ }^{7}$

Therapeutic arterial embolization is a recognized method of controlling hormone production from endocrine tumours of the pancreas and in the liver ${ }^{8,9}$ and has been used less often for embolization of phaeochromocytoma ${ }^{10}$ and carcinoid tumours. ${ }^{11}$ It has not so far been reported in the control of excessive cortisol production from hyperplastic adrenal glands.

We now report the use of bilateral adrenal arterial embolization in the treament of a patient with lifethreatening Cushing's syndrome in whom both medical therapy and percutaneous adrenal venous ablation had failed to effect metabolic or clinical control.

Correspondence: S.B. Blunt, M.R.C.P.

Accepted: 3 March 1989

\section{Case report}

A 51 year old woman presented to a local hospital in 1977 with a swelling in her neck. Calcitonin levels were found to be elevated, and a diagnosis of medullary carcinoma of the thyroid (MCT) was made. The tumour was resected and she received local radiotherapy as there was involvement of one cervical lymph node. In 1983 mediastinal metastases appeared and were treated with local radiotherapy.

Between May 1986 and April 1987 she developed the full picture of severe Cushing's syndrome: she gained $38 \mathrm{~kg}$ in weight, became suicidally depressed and developed severe proximal muscle weakness such that she could not turn over in bed, and she was also found to be diabetic. She was commenced on high doses of both metyrapone ( $750 \mathrm{mg}$ four times a day) and aminoglutethimide ( $250 \mathrm{mg}$ four times a day) with little effect on the high cortisol levels, and the clinical severity of the Cushing's syndrome continued to advance.

In June 1987 she was referred to our centre for further management. Clinically, she was extremely Cushingoid, and appeared very ill. When off all treatment, she had a hypokalaemic alkalosis and fasting hyperglycaemia. Grossly elevated basal values for serum cortisol (with loss of diurnal rhythm), plasma ACTH, and urine free cortisol (UFC) levels were found (Table I). A high dose dexamethasone suppression test failed to produce a fall in the steroid or ACTH levels, and a metyrapone test failed to produce a rise in plasma ACTH. The clinical and biochemical picture thus supported an ectopic source

(C) The Fellowship of Postgraduate Medicine, 1989 
for the ACTH. ${ }^{12}$ This diagnosis was supported by a re-examination of the original MCT tumour tissue which showed positive immunocytochemical staining for the presence of ACTH.

We were thus dealing with a patient whose metastatic MCT was producing ectopic ACTH and severe Cushing's syndrome and who required urgent reduction in cortisol levels if she was to survive. For the MCT, she received a 3-day course of chemotherapy with etoposide $175 \mathrm{mg} /$ day (i.v.) but following this, the plasma calcitonin levels did not fall. Treatment for the Cushing's syndrome at that time was viewed as palliative, as she was clearly unfit for surgical adrenalectomy. Maximal medical therapy with combined metyrapone and aminogluthimide for the first two and a half months of her illness, and metyrapone alone for subsequent periods, had failed to produce a biochemical remission (Figure 1) and clinically there was continuing deterioration. Non-compliance was an unlikely reason for the lack of effect of the medication, as the patient was in hospital for much of the early part of her illness, and her medication was supervised closely by nursing staff. She was a highly motivated and co-operative patient. It was therefore decided that an attempt should be made at bilateral adrenal venous ablation. This was performed in July 1987 under steroid and antibiotic cover.

\section{Method for adrenal infarction and results}

The left adrenal gland was selectively catheterized using a percutaneous approach from the right femoral vein under local anaesthesia. A mixture of $1 \mathrm{ml}$ absolute alcohol and $1 \mathrm{ml}$ iohexol was injected retrogradely into the gland and the vein then occluded with miniature steel coils. On the right side an alcohol/ contrast medium injection was performed but no coils inserted owing to the short length of the adrenal vein. At the end of the procedure there was no demonstrable venous flow from either adrenal gland (Figure 2).

Following this first procedure, the cortisol levels were still elevated and she was recommenced on her previous dose of metyrapone, $3 \mathrm{~g} /$ day. Three weeks later, a second venous ablation was undertaken. This time the procedure was performed on the right side only, as the left was found to be completely occluded. Following this the cortisol levels remained high. It was decided therefore to approach the adrenals from the arterial side, and a bilateral arterial embolization was performed.

Selective arterial studies performed under local anaesthesia from a right femoral approach showed the principal arterial supply to both glands to be from combined superior and middle adrenal arterial trunks

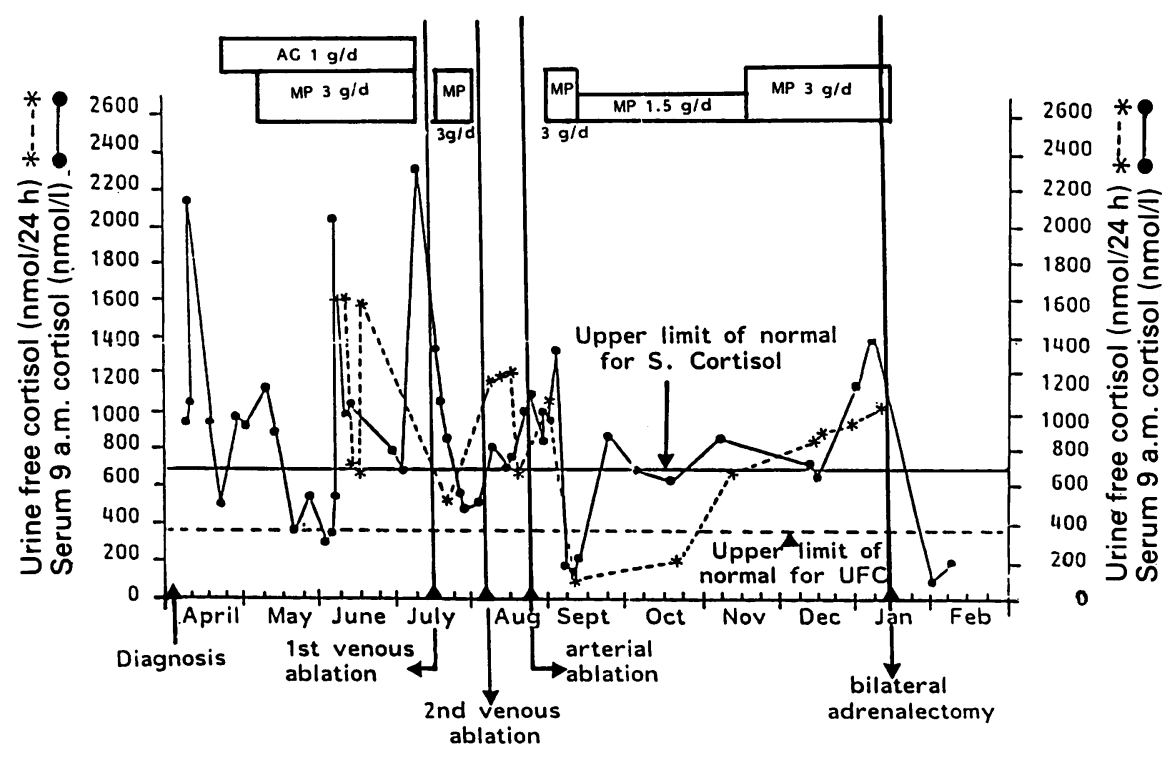

Figure1 Use of two venous and one arterial adrenal ablations to obtain temporary improvement in cortisol levels, and enable eventual surgical adrenalectomy. The period covered is April 1987 to February 1988. 

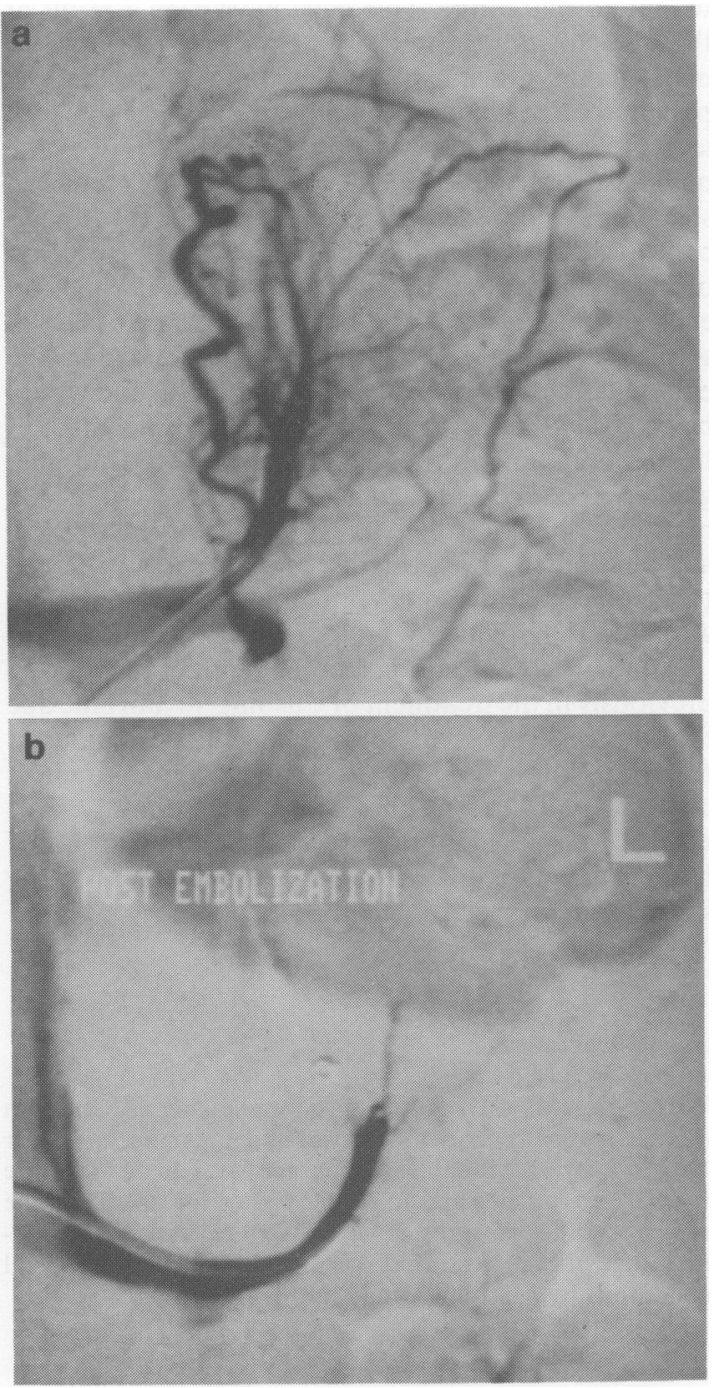

Figure 2 (a) Pre-embolization left adrenal venogram. (b) Left adrenal venogram post-embolization with alcohol, iohexol and coils.

arising from the phrenic arteries. The inferior adrenal arteries were identified as arising conventionally from the renal vessels but both were exceedingly small and apparently supplying only a small area of the lower lobe of the gland on each side. The superior and middle arterial trunks on each side were embolized with finely-divided human dura mater, alcohol and miniature steel coils. At the end of the procedure the arterial flow to the major part of both adrenal glands had been abolished.
Immediately after the arterial embolization, the $09.00 \mathrm{~h}$ cortisol values ranged between $800-1,200 \mathrm{nmol} / \mathrm{l}$. Two weeks after the procedure when the serum cortisol level was $1,200 \mathrm{nmol} / \mathrm{l}$, she was recommenced on her previous dose of metyrapone ( $3 \mathrm{~g} /$ day) and for the first time since diagnosis, the serum and urine cortisol levels fell to within the normal range (Figure 1). On the fourth day of metyrapone, whilst running $09.00 \mathrm{~h}$ serum cortisols of $200-230 \mathrm{nmol} / 1$, she developed clinical features suggestive of hypoadrenalism: she felt generally weak and nauseated and developed postural hypotension. The dose of metyrapone was halved and her symptoms resolved.

She was discharged home on the 21 st day after arterial embolization on metyrapone $1.5 \mathrm{~g} /$ day. Clinically she steadily improved. However, the reduced dose of metyrapone $(1.5 \mathrm{~g} /$ day $)$ was unable to maintain the cortisol levels within the normal range for long, and 3 months following her arterial embolization, the levels began to climb again (Figure 1). An increased dose of metyrapone ( $3 \mathrm{~g} /$ day) made little impact on the cortisol levels which were now rapidly rising.

A second attempt at adrenal arterial embolization was made as there still seemed to be a chance of avoiding major surgery. This proved technically difficult and was abandoned. However, it was felt that she was clinically now a reasonable candidate for surgery. Bilateral adrenalectomy (Mr J.A. Lynn) was performed under steroid cover. Both glands were found to be adherent to surrounding tissue, but were successfully removed. The glands weighed $15 \mathrm{~g}$ (right) and $10 \mathrm{~g}$ (left) (normal weight of one gland is between 3.5 to $5.3 \mathrm{~g}$, and combined weights are 7 to $11 \mathrm{~g}$ ). Microscopically there was uniform cortical hyperplasia with areas of infarction and chronic inflammatory cell infiltration scattered throughout the sections, consistent with the previous ablation procedures. Postoperatively, she made a dramatic improvement in all respects: she became much less Cushingoid, the diabetes and hypertension improved further, and her serum cortisol normalized on hydrocortisone replacement therapy (Figure 1). She is now ambulant at home, 10 months after surgery, and regaining her health. She remains asymptomatic in respect of the MCT.

\section{Discussion}

This patient's condition highlights some management problems that may emerge in the care of a severely Cushingoid patient who has become unfit for surgery, or in whom such treatment is inappropriate, and in whom maximal medical therapy has failed to produce remission. Bilateral adrenal venous ablation was per- 
Table I Corticosteroid and ACTH values off all treatment

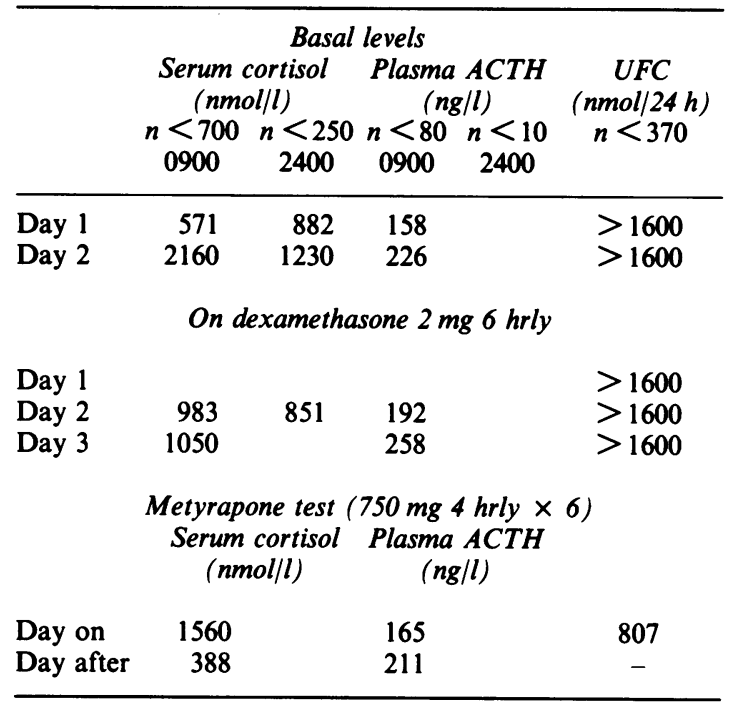

UFC = urinary free cortisol.

formed on two separate occasions but even with the reinstatement of medical therapy, there was an insufficient fall in the cortisol levels and clinically she remained unchanged.

Adrenal arterial embolization was therefore attempted and immediately following this procedure her cortisol levels were still high. This may have reflected release of cortisol from the necrosing glands. Subsequently, on recommencing metyrapone therapy, her cortisol levels were initially easily controlled with a low dose of metyrapone. This was the first time during the patient's illness that serum cortisol levels had fallen

\section{References}

1. Fellerman, H., Dalakos, T.G. \& Streeton, D.H. Remission of Cushing's syndrome after unilateral adrenal phlebography. Apparent destruction of adrenal adenoma. Ann Intern Med 1970, 73: 585-589.

2. Eagon, R.T. \& Page, M.I. Adrenal insufficiency following bilateral adrenal venography. $J A M A$ 1971, 215: 115-116.

3. Fischer, C.E., Turner, F.A. \& Horton, R. Remission of primary hyperaldosteronism after adrenal venography. N Engl J Med 1971, 285: 334-336.

4. Eddy, R.L., McMurray, J., Best, G.B., Henderson, B.W. \& Smith, V.T. Adrenal ablation by venous catheter. Ann Intern Med 1973, 79: 273-274.

5. Rogove, H.J., Meaney, T.F. \& Schumacher, O.P. Cushing's syndrome successfully treated by transcatheter adrenal ablation. Clin Res 1976, 24; 10A. within normal range. This must have been a result of the embolization procedure, as an identical dose of metyrapone previously had had no effect on the patient's clinical condition, nor on the serum cortisol levels. This drug can be remarkably effective in the mild case ${ }^{13}$ However, medical therapy was unable to sustain the reduced cortisol levels, presumably due to continued ACTH-stimulation of the surviving portion of the adrenal gland as the inferior adrenal artery on each side had not been embolized. The rate of rise in serum cortisol levels in the months following the embolization procedure was quite gradual, and would be consistent with progressive escape from control of the remaining un-infarcted portion of the adrenal glands. Nevertheless, the patient was clinically markedly improved: she was mobile, normotensive, and her diabetes was easier to control; she was judged to have been rendered fit enough for surgical bilateral adrenalectomy which was performed successfully.

Adrenal arterial embolization has not been reported previously in the treatment of Cushing's syndrome due to ectopic ACTH. This approach resulted in sufficient reduction in disease activity for metyrapone to effect control, and prepare her for adrenalectomy. We suggest that adrenal arterial embolization might now be considered to be a therapeutic option, at least in the short term, in such patients.

\section{Acknowledgements}

We are grateful to Dr A.J. Munro for allowing us to publish this account of this patient who was under his care, and to $\mathrm{Dr}$ Paul D. Lewis for immunostaining the MCT to demonstrate the ACTH content, and to Dr C. Foster for providing the report on the excised adrenals. We are pleased to acknowledge the successful surgery under difficult technical conditions by Mr John Lynn.

6. Rosenstock, J., Allison, D., Joplin, G.F. et al. Therapeutic adrenal venous infarction in ACTHdependent Cushing's syndrome. Br J Radiol 1981, 54: 912-915.

7. Mathias, C.J., Carron, D., Hemingway, A. \& Allison, D.J. Therapeutic venous infarction of an aldosterone producing adenoma (Conn's tumour). Br Med J 1984, 288: $1416-1417$.

8. Allison, D.J. Therapeutic embolization. Br J Hosp Med 1978, 20: 705-710.

9. Assaad, S.N., Carrasco, C.H., Vassilopoulou-Sellin, R. \& Samaan, N.A. Glucagonoma syndrome: rapid response following arterial embolisation of glucagonoma metastatic to the liver. Am J Med 1987, 82: 533-535. 
10. Horton, J., Hrabovsky, E., Klingberg, W., Hostler, J.A \& Jenkins, J. Therapeutic embolisation of a hyperfunctioning phaeochromocytoma. Am J Radiol 1983, 140: 987-988.

11. Hughes, C.F. Mediastinal carcinoid tumour: preoperative arterial embolisation. Aust NZ J Med 1986, 16: 808-810.
12. Liddle, G.W. Cushing's syndrome. In: Eisenstein, A.B. (ed.) The Adrenal Cortex, edition 1. Little, Brown \& Co., Boston, 1967, pp. 523-551.

13. Donckier, J., Burrin, J.M., Ramsay, I.D. \& Joplin, G.F. Successful control of Cushing's disease in the elderly with long term metyrapone. Postgrad Med J 1986, 62: 727-730. 